\title{
Osteoid Osteoma of the Mandible - Clinical and Histological Findings
}

\author{
LEVI MATTHIES ${ }^{1 *}$, TIM ROLVIEN ${ }^{2,3^{*}}$, THOMAS J. PAKUSA ${ }^{4}$, CHRISTIAN KNIPFER ${ }^{1}$, \\ MARTIN GOSAU ${ }^{1}$, MICHAEL AMLING ${ }^{2}$, REINHARD E. FRIEDRICH ${ }^{1}$ and JOZEF ZUSTIN ${ }^{2,5}$ \\ ${ }^{1}$ Department of Oral and Maxillofacial Surgery, \\ University Medical Center Hamburg-Eppendorf, Hamburg, Germany; \\ ${ }^{2}$ Department of Osteology and Biomechanics, University Medical Center Hamburg-Eppendorf, Hamburg, Germany; \\ ${ }^{3}$ Department of Orthopedics, University Medical Center Hamburg-Eppendorf, Hamburg, Germany; \\ ${ }^{4}$ MKG Falkenried, Hamburg, Germany; \\ ${ }^{5}$ Pathologie Hamburg, Hamburg, Germany
}

\begin{abstract}
Background/Aim: Osteoid osteoma $(\mathrm{OO})$ is a benign tumor characterized by the excessive formation of unmineralized bone matrix. It is mostly located in the long bones of the lower extremities or the spine, but can also occur in atypical locations. Patients and Methods: Here we report on a rare case of $\mathrm{OO}$ in the mandible in an 18-year-old male patient with a simultaneous finding of a pilomatrixoma in the parietal region. Initially, a biopsy had been taken due to an unclear tumor mass and non-specific pain adjacent to tooth 46. Results: The detailed radiographic and histopathological analysis revealed the diagnosis of $\mathrm{OO}$ in terms of a characteristic radiolucent nidus and structurally disorganized woven bone with osteoid, respectively. The tumor was subsequently completely resected resulting in a complete recovery of symptoms. Conclusion: As we demonstrate an infrequent finding of $\mathrm{OO}$ in the mandible, it is important to diagnose and treat this condition appropriately.
\end{abstract}

Osteoid osteoma (OO) is a benign bone tumor producing unmineralized bone called osteoid. It mostly affects adolescents and young adults and is typically located in the long bones of the femur and tibia as well as the vertebra, but various locations have been described (1). OO accounts for approximately $10 \%$ of all benign bone tumors and is more

*These two Authors contributed equally to this work.

Correspondence to: Tim Rolvien, MD, Department of Osteology and Biomechanics, University Medical Center Hamburg-Eppendorf, Lottestr. 59, 22529 Hamburg, Germany. Tel: +49 40741056373, e-mail: t.rolvien@uke.de

Key Words: Osteoid osteoma, mandible, bone tumor, histology, cone beam $\mathrm{CT}$. commonly found in males (2). It has as a centrally located small, oval or round area called nidus, which is composed of osteoid and trabeculae of newly-formed bone deposited within a substratum of highly vascularized osteogenic connective tissue and a peripheral zone of sclerosis. The predominant clinical feature is localized pain, typically nocturnal and responsive to nonsteroidal anti-inflammatory drugs (NSAIDs), especially salicylates. This can be found in approximately $80 \%$ of the cases and is most likely due to prostaglandin production and expression of cyclooxygenase (COX) (3).

Cases of $\mathrm{OO}$ of the mandible have recently been reported, but remain a very rare finding (4). Previous reviews were able to identify around 20 published cases $(4,5)$. In this case report, we demonstrate the clinical and micro-morphological manifestation of this benign osseous tumor located in the right lower jaw.

\section{Case Report and Results}

History. A healthy 18-year-old male patient presented to a private practice for oral and maxillofacial surgery in spring 2018 due to persistent and non-specific, particularly nocturnal pain, alleviated by NSAIDs. The pain was located in the right lower jaw, non-radiating and of dull quality. The patient had first noticed this pain, severe and intermittent, aggravated by palpation, a few months earlier. The initial panoramic dental radiograph showed retained teeth 18, 28 and 48 (Figure 1A). An additional dental CT indicated an unclear tumor mass near the mesial root of tooth 46 extending $0.9 \times 0.8 \times 0.5 \mathrm{~cm}^{3}$ (Figure 1B-D). Due to this unclear tumor mass and in combination with the reported pain, the wisdom teeth were extracted and an additional biopsy of the region of interest was made. The oral mucosa above the tumor mass appeared clearly reddened. The resection specimen showed woven bone trabeculae covered 
by enlarged osteoblasts and multinucleated osteoclasts and embedded in fibrous stroma containing dilated blood vessels (Figure 2A and B). From this, the histopathologic diagnosis of $\mathrm{OO}$ was concluded.

Physical examination. With this diagnosis, the patient was referred to the University Medical Center HamburgEppendorf, Germany. On clinical examination, a scar of the lingual mandibular mucosa was noticeable several weeks after probe incision in the lower right molar region. The intact overlying mucosa did not raise suspicion for inflammation, redness or swelling. The tooth 46 was not found to be sensitive to percussion, not mobile and dental pulp testing was positive, as were the adjacent teeth. Cervical lymph nodes were not enlarged or tender to palpation. An additional finding was a movable swelling of firm elastic consistency in the central parietal region of the head. The patient was unaware of the course of progression, as this was not causing any pain or discomfort.

Diagnosis and treatment. A panoramic radiograph taken prior to the initial biopsy indicated an inhomogeneous translucency in the first molar region of the lower right jaw (Figure 3A). An additional cone beam CT with increased bone resolution and greater overview revealed a nidus with a diameter of $7.6 \mathrm{~mm}$ (Figure $3 \mathrm{~A}-\mathrm{D}$ ). Both the cone beam $\mathrm{CT}$ and the previously performed dental $\mathrm{CT}$ showed a central zone of a radiodense mineralized tissue that was distinguishable from a peripheral zone of radiolucency, again surrounded by a zone of dense sclerotic bone.

After completion of diagnosis the patient underwent general anesthesia to have the benign osseous tumor surgically removed, with simultaneous excision of the parietal formation. To this end, an incision of the lingual mucosa was made from tooth 43 to 47 . A mucoperiosteal flap was formed. After subperiosteal preparation, an osteotomy in the region of 4546 was done with a dental drill. After visualization of the spongy bone tumor it was removed by excochleation. The immediately adjacent inferior alveolar nerve as well as the mesial root of tooth 46 were preserved. Bone wax was instilled to facilitate hemostasis and healing of the bony defect. The mucoperiosteal flap was readapted and the wound defect thoroughly closed. The parietal tumor was resected by spindle-shaped incision in full depth to the epicranial aponeurosis. After thorough hemostasis the wound defect was primarily closed.

Histologic findings. During the course of the surgery, two specimens were fixed in buffered formalin and sent for histopathological evaluation. Following an EDTAdecalcification of the surgical specimen (maximum diameter $0.8 \mathrm{~cm}$ ), a subsequent histological analysis confirmed the diagnosis of OO. The tumor harvested intraorally consisted of structurally disorganized woven bone with nonmineralized osteoid and prominent vasculature (Figure 4A). A central zone of osteosclerosis could be distinguished from a peripheral zone with less mature cells and little matrixformation. The cells were characterized by an osteoblastic disorganized overgrowth with the formation of immature bone as well as scattered multinucleated osteoclasts (Figure 4B). The tumor had formed a fibroblastic pseudo capsule. No signs of malignancy were noted. Measurements were $0.8 \times 0.5 \times 0.5 \mathrm{~cm}$. The parietal tumor was found to be a regressive pilomatrixoma, excised in toto (not shown). Measurements were $1.6 \times 0.5 \times 1.0 \mathrm{~cm}^{3}$.

Follow-up. After quick recovery and an uneventful course, the patient was discharged from the hospital on postoperative day one. The panoramic radiograph taken postoperatively showed no signs of fracture, dental damage or displacement. Furthermore, there were no signs of a remaining tumor mass (Figure 5A). The patient presented to our outpatient clinic three months after surgery. An additional cone beam $\mathrm{CT}$ confirmed the complete removal of the tumor (Figure 5B-D). The intraoral wound healing showed good consolidation. Teeth 45, 46 which were immediately adjacent to the OO showed no signs of mobility, pulp test was positive. The patient was completely pain-free and adhering to regular follow-up.

\section{Discussion}

In the present study, a very rare finding of osteoid osteoma (OO) of the mandible including a detailed radiographic and histopathological documentation was demonstrated. This entity was first described in 1935 by Jaffe (6). About $80 \%$ are located in the tibia, femur or spine, and less than $1 \%$ in the jaws (7). Of the few existing reports on jaw OO, there appears to be a predilection for the mandible rather than the maxilla, which is in line with this report (8). Taking into account the very few reports, jaw OO did not show a genderspecific epidemiology, as opposed to other anatomic locations (2).

For appropriate treatment, precise diagnostic localization and classification of the lesion are essential. The initial differential diagnoses included osteoid osteoma, osteoblastoma, cementoblastoma, sclerosing osteomyelitis or others, such as a residual cyst, complex odontoma, ossifying fibroma or idiopathic osteosclerosis. Considering the diagnostic options, cone-beam CT imaging represents a suitable technique to detect $\mathrm{OO}$ as previously shown for CT and other locations, with increased bone resolution and decreased radiation exposure (9-11). In fact, cone beam CT is particularly valuable in the detection of $\mathrm{OO}$ as the nidus may be very small and therefore not detectable in normal radiographs or CT scans (12). In our case, the cone beam CT 


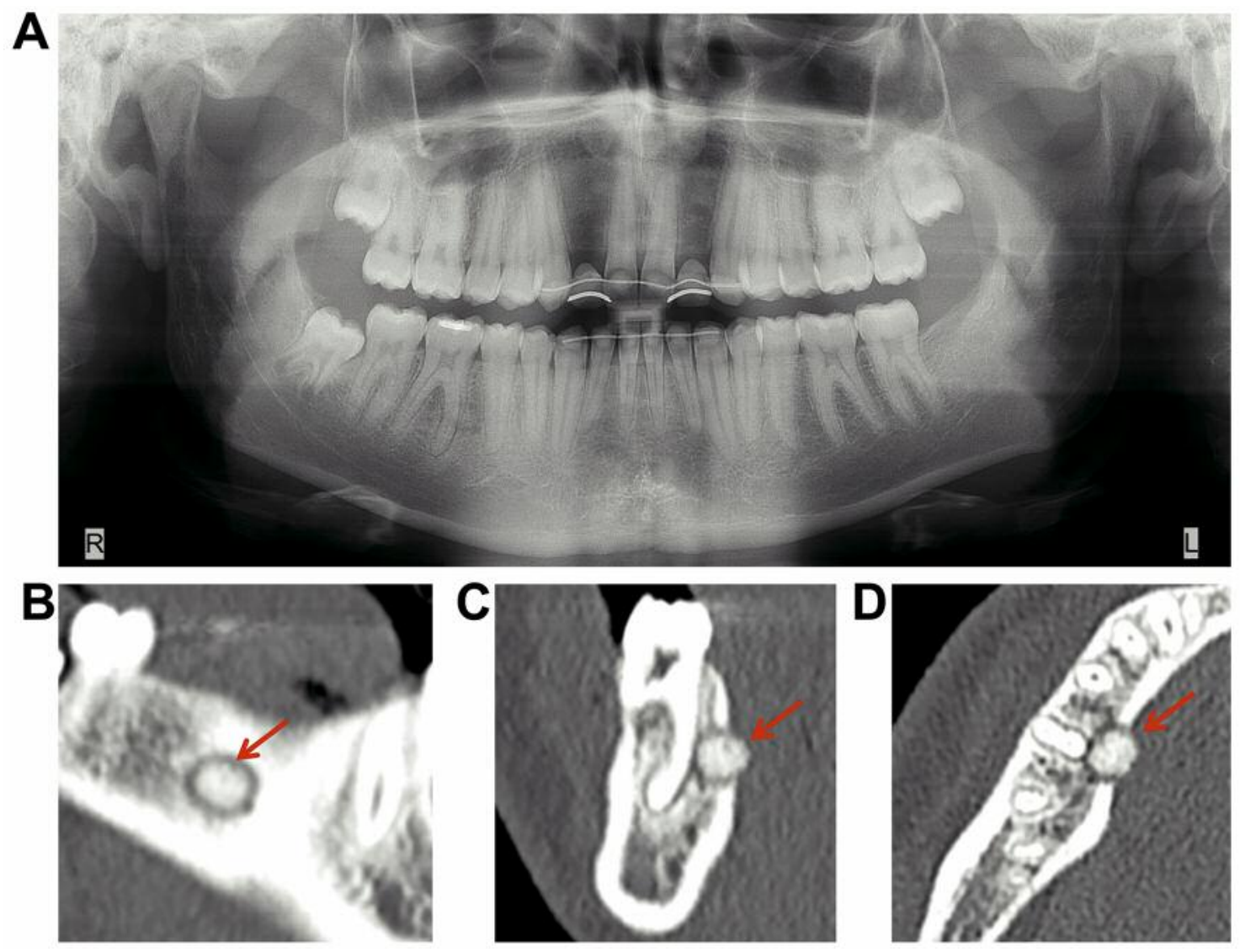

Figure 1. Preoperative imaging. (A) Panoramic radiograph taken before the wisdom tooth extraction and probe excision. Note the retained tooth 48 as a possible source of complaint. Teeth 12 and 22 are missing, a bridge is in place. Dental CT is displaying the tumor in (B) sagittal (C) coronal and $(D)$ axial slicing. Red arrows indicate the structure of interest.
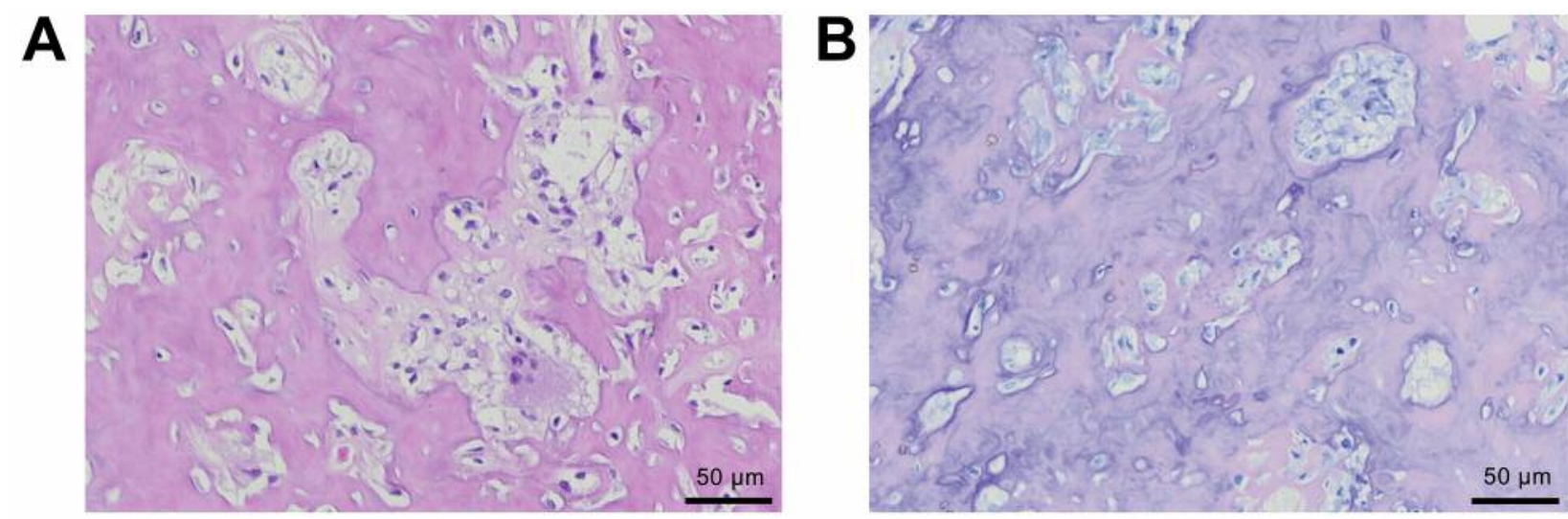

Figure 2. Histological findings in the first biopsy. (A) Woven bone trabeculae covered by enlarged osteoblasts and scattered multinucleated osteoclasts can be seen. The fibrous stroma contains dilated blood vessels. $H \& E$ staining. (B) In the central part of the specimen, the trabeculae appear as a an almost cortical structure reflecting the central mineralization seen in the CT. Giemsa staining. EDTA decalcification.

revealed a central mineralization that was distinguishable from a peripheral radiolucency, again surrounded by a zone of dense sclerotic bone. This clearly corresponds to the radiological description of $\mathrm{OO}$.
Although the clinical, radiographic and histological findings of OO and osteoblastoma overlap $(13,14)$, the WHO classification defines $\mathrm{OO}$ as a distinct entity with neoplastic properties and limited expansion, resulting in lesions with a 

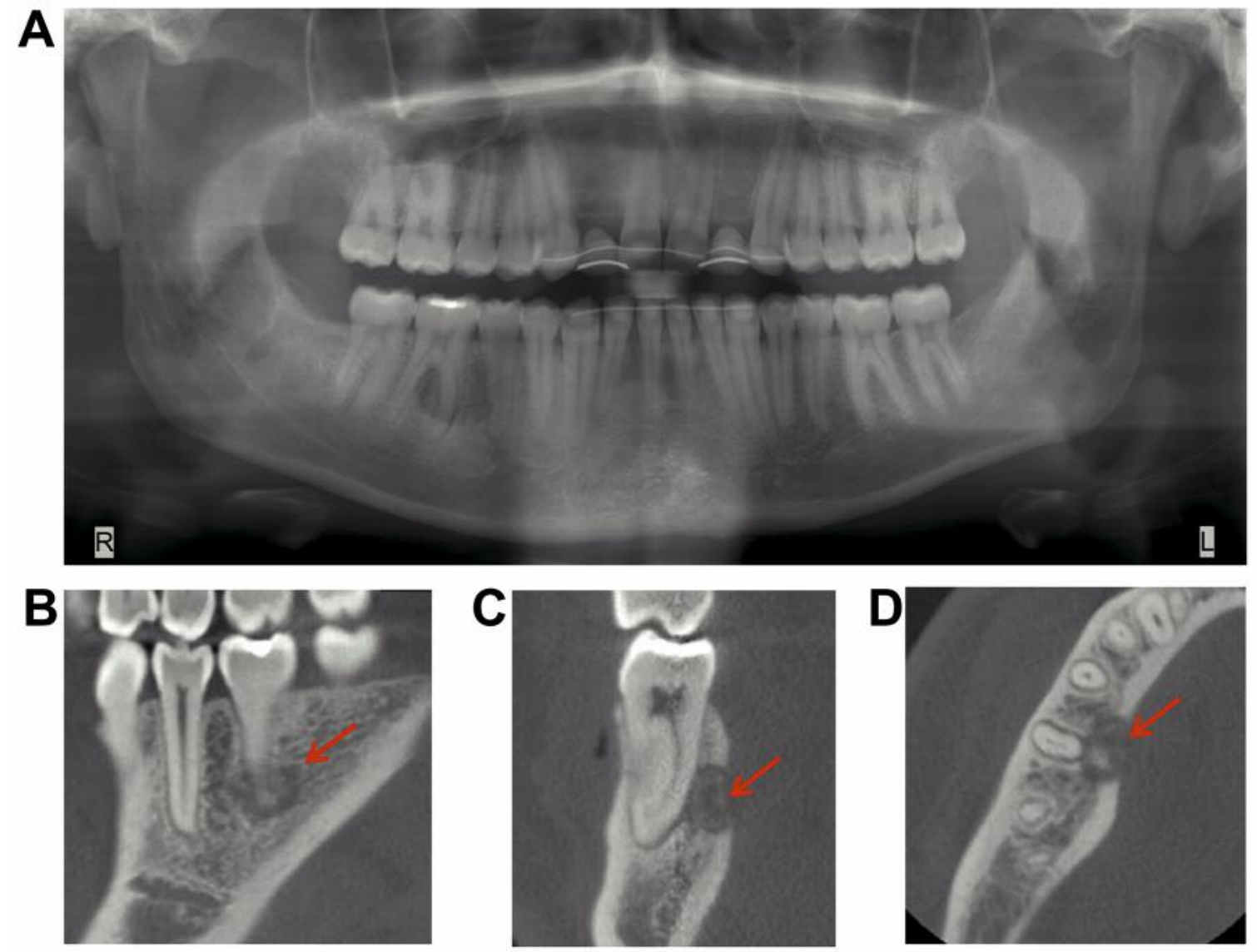

Figure 3. Postoperative radiograph and $(B-D)$ cone beam $C T$ displaying the radiolucent tumor with remaining central mineralization in $(B)$ sagittal (C) coronal and (D) axial slicing. Red arrows indicate the structure of interest.
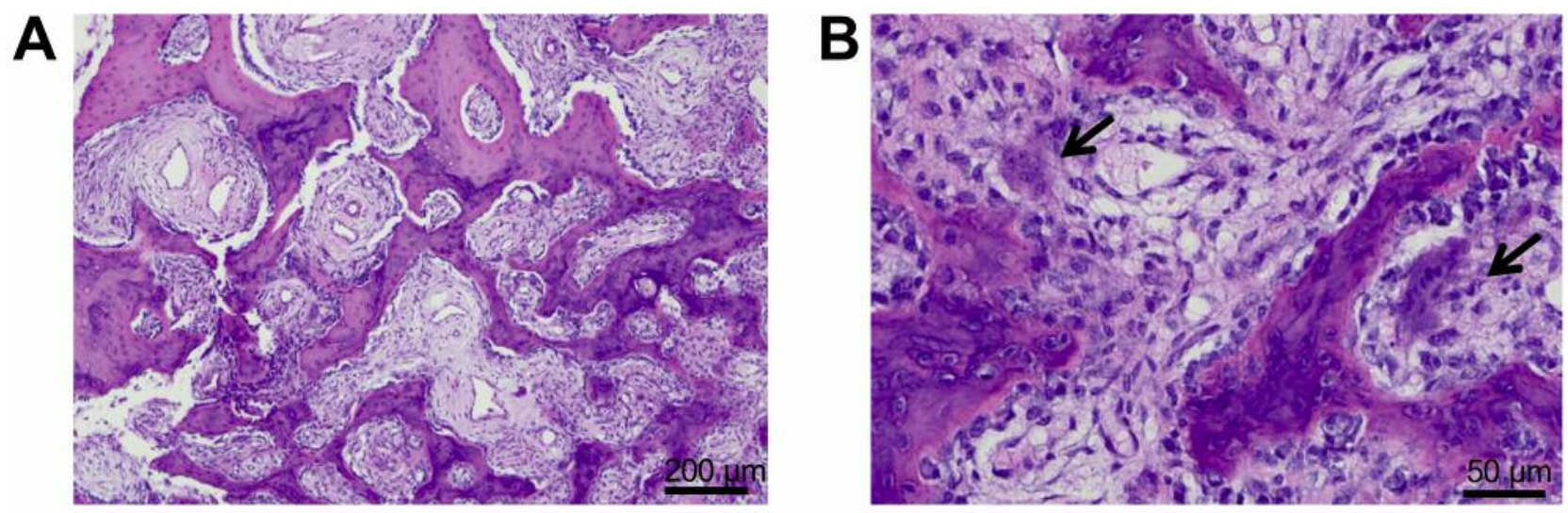

Figure 4. Detailed histological examination of the resection specimen. (A) Overview. (B) Scattered multinucleated osteoclasts (black arrows) and woven bone trabeculae can be seen in higher magnification. $H \& E$ staining. EDTA decalcification.

diameter less than $2 \mathrm{~cm}(15)$. Therefore, some of the previously reported cases are more likely osteoblastomas due to their size $>2 \mathrm{~cm}$, indicating that the overall number of true $\mathrm{OO}$ within the jaw may be even lower than 20 cases $(16,17)$. Several reports underline the similarities between $\mathrm{OO}$ and osteoblastoma. Common features are areas of lesional nonosteoblastic stroma, presence of scattered, large cells with degenerate nuclei and innervation, ultimately suggesting 

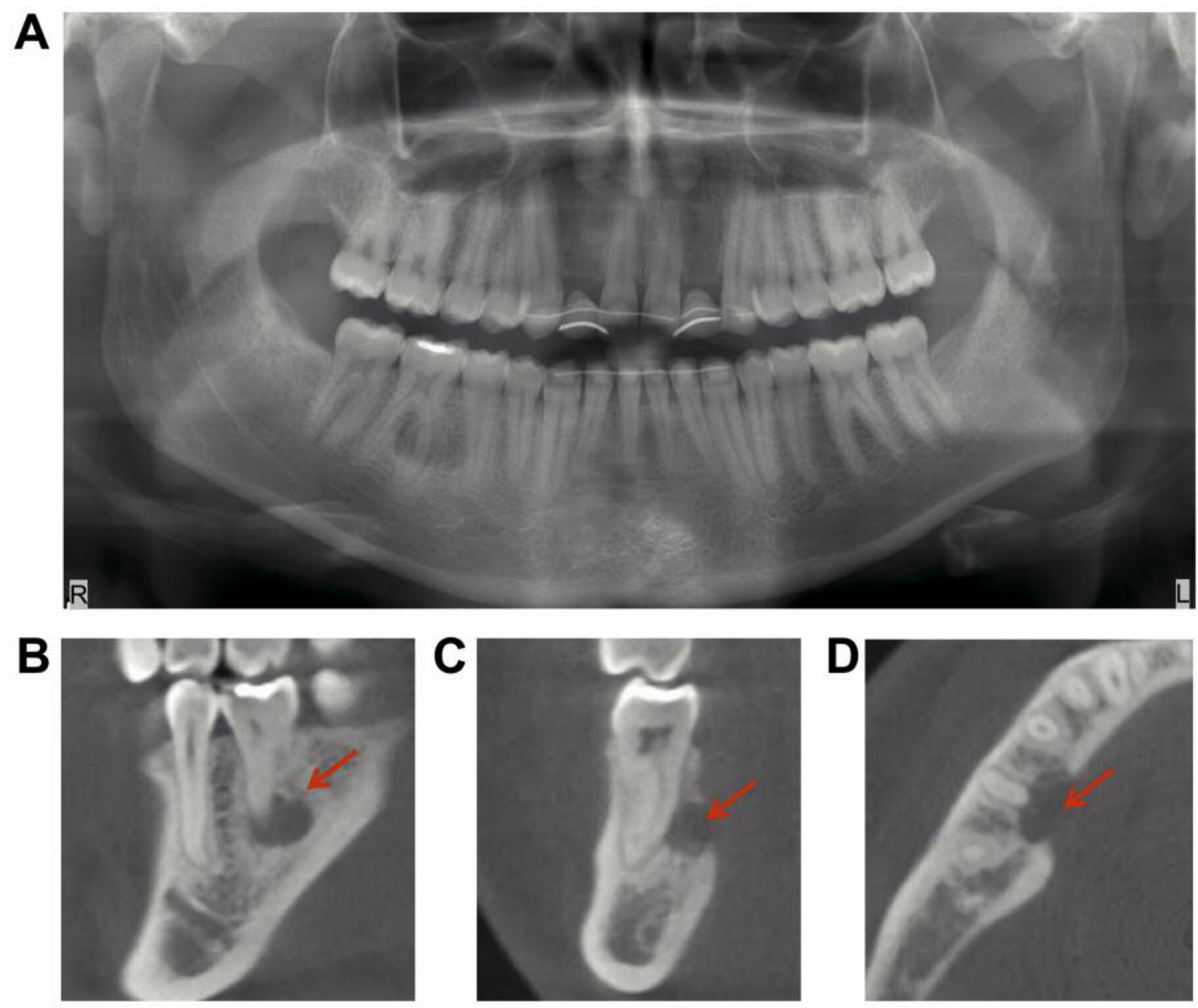

Figure 5. Imaging performed three months postoperatively. (A) Panoramic radiograph taken after excochleation of the tumor. Note the osseous translucency adjacent to teeth 45/46. (B-D) Cone beam CT (sagittal, coronal and axial slicing) confirms complete resection of the tumor.

classification of both as a single entity (18). However, beyond the size criterion, $\mathrm{OO}$ is classically localized in the long bones and may exhibit a stronger sclerotic reaction compared to osteoblastoma, which is rather located in the axial bones (19). Further analysis of compounds such as osteocalcin, released by OO, may be additionally used to differentiate the tumor (20).

In our case, we could demonstrate the histological characteristics of OO including woven bone, multinucleated osteoclasts and a fibrous stroma with enlarged blood vessels in both specimens. However, the surgical removal of the OO allowed an appropriate histological analysis and confirmation of the correct diagnosis. To this end, the specimen required undergoing thorough decalcification. Regrettably, subsequent visualization of osteoid accumulation or innervation via immunohistochemistry was not feasible.

In fact, when the $\mathrm{OO}$ can be excised in toto, the patient will most likely be pain-free after surgery, with an overall low rate of recurrence in the majority of cases $(21,22)$. Radiofrequency ablation as an alternative mode of treatment was not chosen in our case given that the site was surgically accessible, with justifiable risk of damage to surrounding tissues. Furthermore, percutaneous treatment methods although less invasive do not provide histological specimens, which may aid in differential diagnosis (23). However, both techniques show comparable results with regards to recurrence (24).

Concurrent findings of osteomas and pilomatrixoma may be associated with Gardner's Syndrome (Familial colorectal polyposis, FAP) (25). FAP is due to an autosomal dominant genetic loss-of-function mutation of the $A P C$ gene, resulting in activation of the canonical Wnt-signaling pathway. Clinical findings most prominently include colonic polyps that can undergo malignant transformation as well as extracolonic tumors. As family history in this patient was not significant and there were no clinical gastrointestinal ailments and an overall low index of suspicion, further genetic diagnostics or colonoscopy were as of now neglected. 
Taken together, we outlined the diagnosis, treatment and histological features in a rare case of osteoid osteoma of the mandible. Due to the very low number of cases with OO of the jaw, it is important for dentists, oral and maxillofacial surgeons to report additional cases to increase the awareness and potentially diagnose and appropriately treat this condition at the earliest possible.

\section{References}

1 Rolvien T, Zustin J, Mussawy H, Schmidt T, Pogoda P and Ueblacker P: Intra-articular osteoid osteoma as a differential diagnosis of diffuse mono-articular joint pain. BMC Musculoskelet Disord 17(1): 455, 2016.

2 Atesok KI, Alman BA, Schemitsch EH, Peyser A and Mankin H: Osteoid osteoma and osteoblastoma. J Am Acad Orthop Surg 19(11): 678-689, 2011.

3 Mungo DV, Zhang X, O'Keefe RJ, Rosier RN, Puzas JE and Schwarz EM: COX-1 and COX-2 expression in osteoid osteomas. J Orthop Res 20(1): 159-162, 2002.

4 Singh A and Solomon MC: Osteoid osteoma of the mandible: A case report with review of the literature. J Dent Sci 12(2): 185189, 2017.

5 An SY, Shin HI, Choi KS, Park JW, Kim YG, Benavides E, Kim JW and An CH: Unusual osteoid osteoma of the mandible: Report of case and review of the literature. Oral Surg Oral Med Oral Pathol Oral Radiol 116(2): e134-140, 2013.

6 Jaffe HL: "Osteoid-osteoma": A benign osteoblastic tumor composed of osteoid and atypical bone. Arch Surg 31(5): 709$728,1935$.

7 Ida M, Kurabayashi T, Takahashi Y, Takagi M and Sasaki T: Osteoid osteoma in the mandible. Dentomaxillofac Radiol 31(6): 385-387, 2002.

8 Rahsepar B, Nikgoo A and Fatemitabar SA: Osteoid osteoma of subcondylar region: Case report and review of the literature. J Oral Maxillofac Surg 67(4): 888-893, 2009.

9 Assoun J, Richardi G, Railhac JJ, Baunin C, Fajadet P, Giron J, Maquin P, Haddad J and Bonnevialle P: Osteoid osteoma: MR imaging versus CT. Radiology 191(1): 217-223, 1994.

10 Harun M, Hayrettin Y, Serhat M, Engin C, Kamil C, Armagan A and Sancar PA: Atypical location of an osteoid osteoma with atypical anterior knee pain. Int J Surg Case Rep 5(11): 873-876, 2014.

11 Pikoulas C, Mantzikopoulos G, Thanos L, Passomenos D, Dalamarinis C and Glampedaki-Dagianta K: Unusually located osteoid osteomas. Eur J Radiol 20(2): 120-125, 1995.

12 Chaudhary M and Kulkarni M: Osteoid osteoma of mandible. J Oral Maxillofac Pathol 11(2): 52, 2007.

13 Alvares Capelozza A, Gião Dezotti M, Casati Alvares L, Negrão Fleury R and Sant'Ana E: Osteoblastoma of the mandible: Systematic review of the literature and report of a case. Dentomaxillofac Radiol 34(1): 1-8, 2005.
14 Rawal YB, Angiero F, Allen CM, Kalmar JR, Sedghizadeh PP and Steinhilber AM: Gnathic osteoblastoma: Clinicopathologic review of seven cases with long-term follow-up. Oral Oncol 42(2): 123-130, 2006.

15 Christopher D, Fletcher JA and Bridge P: WHO classification of tumours of soft tissue and bone. International agency for research on cancer 4th edition Lyon, pp. 110-111, 2013.

16 Jones AC, Prihoda TJ, Kacher JE, Odingo NA and Freedman PD: Osteoblastoma of the maxilla and mandible: A report of 24 cases, review of the literature, and discussion of its relationship to osteoid osteoma of the jaws. Oral Surg Oral Med Oral Pathol Oral Radiol Endod 102(5): 639-650, 2006.

17 Yang C and Qiu WL: Osteoid osteoma of the eminence of the temporomandibular joint. Br J Oral Maxillofac Surg 39(5): 404406, 2001.

18 Barlow E, Davies A, Cool W, Barlow D and Mangham D: Osteoid osteoma and osteoblastoma: Novel histological and immunohistochemical observations as evidence for a single entity. J Clin Pathol 66(9): 768-774, 2013.

19 Chotel F, Franck F, Solla F, Dijoud F, Kohler R, Berard J, Genevois KA and Group FSG-BTS: Osteoid osteoma transformation into osteoblastoma: Fact or fiction? Orthop Traumatol Surg Res 98(6): S98-S104, 2012.

20 Confavreux C, Borel O, Lee F, Vaz G, Guyard M, Fadat C, Carlier M-C, Chapurlat R and Karsenty G: Osteoid osteoma is an osteocalcinoma affecting glucose metabolism. Osteoporos Int 23(5): 1645-1650, 2012.

21 Kransdorf M, Stull M, Gilkey F and Moser Jr R: Osteoid osteoma. Radiographics 11(4): 671-696, 1991.

22 Kitsoulis P, Mantellos G and Vlychou M: Osteoid osteoma. Acta Orthop Belg 72(2): 119, 2006.

23 Peyser A, Applbaum Y, Khoury A, Liebergall M and Atesok K: Osteoid osteoma: Ct-guided radiofrequency ablation using a water-cooled probe. Ann Surg Oncol 14(2): 591-596, 2007.

24 Weber MA, Sprengel SD, Omlor GW, Lehner B, Wiedenhöfer B, Kauczor HU and Rehnitz C: Clinical long-term outcome, technical success, and cost analysis of radiofrequency ablation for the treatment of osteoblastomas and spinal osteoid osteomas in comparison to open surgical resection. Skeletal Radiol 44(7): 981-993, 2015.

25 Gardner EJ: A genetic and clinical study of intestinal polyposis, a predisposing factor for carcinoma of the colon and rectum. Am J Hum Genet 3(2): 167-176, 1951.
Received November 22, 2018

Revised December 4, 2018

Accepted December 5, 2018 